\title{
A Binary-Induced Pinwheel Outflow from the Extreme Carbon Star, AFGL 3068
}

\author{
Mark Morris ${ }^{1}$, Raghvendra Sahai $^{2}$, Keith Matthews ${ }^{3}$, Judy Cheng ${ }^{4}$, \\ Jessica $\mathbf{L u}^{1}$, Mark Claussen ${ }^{5}$ and Carmen Sánchez-Contreras ${ }^{6}$ \\ ${ }^{1}$ Dept. of Physics \& Astronomy, University of California, Los Angeles, CA 90095-1547, USA \\ email: morris@astro.ucla.edu \\ ${ }^{2}$ Jet Propulsion Laboratory/Calif. Inst. of Technology, MS 183-900, Pasadena, CA 91109, USA \\ ${ }^{3}$ California Institute of Technology, Mail Code 105-24, Pasadena, CA 91125, USA \\ ${ }^{4}$ Dept. of Physics \& Astronomy, The Johns Hopkins Univ., Baltimore, MD 21218, USA \\ ${ }^{5}$ National Radio Astronomy Observatory, 1003 Lopezville Road, Socorro, NM 87801, USA \\ ${ }^{6}$ Dpto. de Astrofísica Molecular e Infrarroja, IEM-CSIC, Serrano 121, 28006 Madrid, Spain
}

\begin{abstract}
The extreme carbon star, AFGL 3068, is losing mass at a rate in excess of $10^{-4}$ $\mathrm{M}_{\odot} \mathrm{yr}^{-1}$, and has so far been detected only in the infrared because it is hidden by a thick dust photosphere having a color temperature of $\sim 300 \mathrm{~K}$. Using the ACS camera on HST, we have imaged AFGL 3068 with broad-band filters at 0.6 and $0.8 \mu \mathrm{m}$ and find a thin, apparently continuous spiral arc winding 4 or 5 times around the location of the star, from angular radii of 2 to 10 arcsec. We interpret this as the projection of nested spiral shells such as were predicted to occur when the mass-losing star is a member of a binary system. In this case, the illumination is presumably provided by ambient galactic starlight. Subsequent near-IR observations with the NIRC2 camera on the Keck II telescope using adaptive optics reveal that AFGL 3068 has two components separated by 0.11 arcsec, or $109 \mathrm{AU}$ at a distance of $1 \mathrm{kpc}$. One very red component is presumably the mass-losing carbon star, while the other component is apparently a much bluer companion. Assuming each component has mass $\mathrm{M}\left(\mathrm{M}_{\odot}\right)$, and ignoring the projection of the separation vector, we find the binary period to be $810 \mathrm{M}^{-0.5}$ yrs, strikingly comparable to the 710-yr separation of the shells obtained from the known outflow velocity of $14.7 \mathrm{~km} \mathrm{~s}^{-1}$.
\end{abstract}

Keywords. stars: AGB and post-AGB, stars: carbon, binaries: general, stars: winds, outflows

A progression of quasi-concentric shells has been observed around a number of preplanetary nebulae (e.g., Sahai et al. 1998; Su 2004), with a characteristic spacing corresponding to time intervals of hundreds of years. No intrinsic mechanism to produce mass loss variations on such a time scale has been identified, but Harpaz et al. (1997) raise the possibility that a binary companion in an eccentric orbit might be responsible. Our HST observations (figure 1) show that AFGL 3068 has a more continuous set of such shells than in any other system, and that these shells are arranged in a spiral pattern. Such a pattern would result naturally from a binary system (Mastrodemos \& Morris 1999), and indeed, our observations with Keck II reveal that this extreme, mass-losing carbon star (e.g., Winters et al. 1997) has a closely-spaced binary companion (figure 2). The binary separation in this system matches that needed to account for the shell spacings, with allowance for projection and assuming stellar masses of $1-2 \mathrm{M}_{\odot}$. The Archimedean spiral form of the shell is well modeled with a circular orbit.

The structure of the AFGL 3068 envelope raises the possibility that binary companions are responsible for quasi-concentric shells in most or all of the systems in which they have been observed. The lack of azimuthal continuity in the shells elsewhere can perhaps 


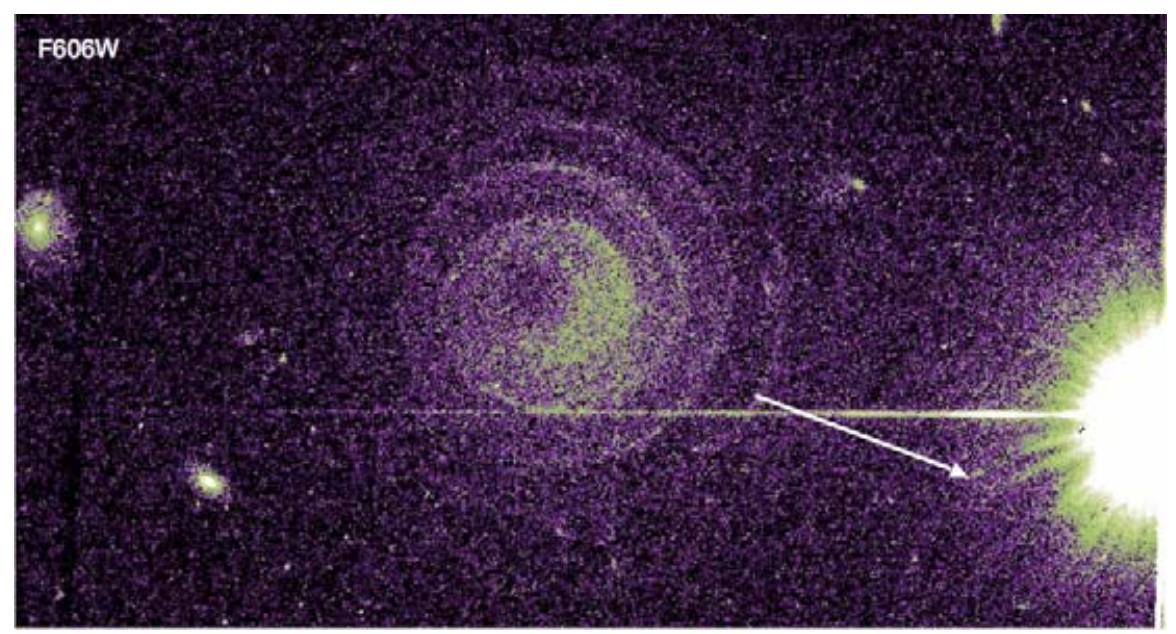

Figure 1. HST/ACS image of AFGL 3068 with the F606W filter (central wavelength $5907 \AA$ ). The dimensions are 50"x27". A similar result was obtained with the F814W filter. The bright star at the right was used as the natural guide star for the Keck AO images shown in figure 2 . The arrow shows the direction of the Galactic plane, $650 \mathrm{pc}$ away for a source distance of $1 \mathrm{kpc}$. We propose that the illumination of the shells is by Galactic starlight. [These data have been separately discussed by N. Mauron \& P.J. Huggins in a recent preprint, astro-ph/0602623.]

Figure 2. Keck adaptive optics images of AFGL 3068 in 3 near-IR filters. Two sources are apparent, the slightly diffuse one to the west probably being the highly obscured, mass-losing star, and the one to the east being a much less obscured companion at a projected distance of $109 \mathrm{AU}$ at $1 \mathrm{kpc}$. The first Airy ring in the point spread function for these images has a trefoil pattern which is most evident in the $\mathrm{H}$ image of the eastern source. (The "PAH" filter was used to provide a narrow-band sampling of the $3.3 \mathrm{~m}$ continuum, since the usual wideband filters saturated on this strong source.)

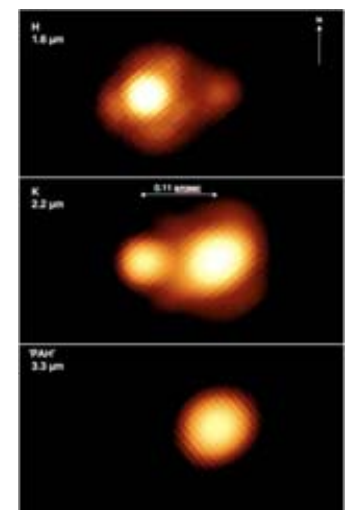

be ascribed to orbital eccentricity, to different projections of the orbital planes, and to unfavorable illumination geometries.

\section{Acknowledgements}

This research is supported by a NASA LTSA award to JPL and UCLA (RTOP grant no. 399-20-40-06). RS thanks NASA for support from awards GO-09463 and GO-10185 from STScI.

\section{References}

Harpaz, A., Rappaport, S. \& Soker, N. 2002, ApJ 487, 809

Mastrodemos, N. \& Morris, M. 1999, ApJ 523, 357

Sahai, R., Trauger, J.T., Watson, A.M., Stapelfeldt, K.R. et al. 1998, ApJ 493, 301

Su, K.Y.L. 2004 in: M. Meixner, J.H. Kastner, B. Balick \& N. Soker (eds.), Asymmetrical Planetary Nebulae III, ASP Conf. Proc. \#313, p. 225

Winters, J.M., Fleischer, A.J., Le Bertre, T. \& Sedlmayr, E. 1997, A\&A 326, 305 